\title{
Expectativas sobre la transición desde la educación básica a la educación media de estudiantes provenientes de contextos de vulnerabilidad social
}

\author{
Expectations on the Transition From Primary to \\ Secondary Education for Students From Contexts of \\ Social Vulnerability
}

\section{Gabriela Gómez Vera, Marlene Rivas Muena, y Constanza Lobos Guerrero}

Universidad de O'Higgins, Chile.

\section{Resumen}

\begin{abstract}
Este estudio aborda las perspectivas de jóvenes, familiares, docentes y directivos que acompañan el proceso de transición desde la educación primaria a la secundaria de estudiantes de octavo año básico en establecimientos de alta vulnerabilidad social. Se analizan el rol y las expectativas de los actores que acompañan esta transición, así como las expectativas de los propios jóvenes y sus familias. Se entrevistaron 32 jóvenes, así como las madres de 12 de ellos, docentes y directivos. Los análisis muestran que, más allá de su rendimiento, todos los estudiantes esperan completar la educación secundaria e integrarse exitosamente en el mundo laboral. El referente casi exclusivo de los jóvenes son sus familias; los docentes y otros actores del establecimiento escolar están ausentes de sus relatos. Los directivos y docentes, por su parte, tienen bajas expectativas respecto del futuro educativo de los estudiantes de octavo ańo, y consideran que se trata de un nivel en el que tienen pocas posibilidades de impactar. En este contexto, los jóvenes viven la transición a la educación media con escaso acompańamiento por parte de los establecimientos, lo que, sumado a las bajas expectativas, supone una situación que pone en riesgo el éxito de esta transición.
\end{abstract}

Palabras clave: transiciones educativas; trayectorias educativas; educación primaria; educación secundaria; expectativas.

Correspondencia a:

Gabriela Gómez Vera

Alameda Libertador General Bernardo O’Higgins 611, Universidad de O'Higgins,

Rancagua, Chile.

gabriela.gomez@uoh.cl

Financiamiento asociado: Proyecto Fondecyt iniciación 11150209 (ANID, Gobierno

de Chile).

(C) 2021 PEL, http://www.pensamientoeducativo.org - http://www.pel.cl

ISSN:0719-0409ＤDI:203.262, Santiago, Chile doi: 10.7764/PEL.58.1.2021.1 


\begin{abstract}
This article examines the perspectives of young people, family members, teachers, and principals that support the process of transition from primary to secondary education for eighth-grade students in schools with high levels of social vulnerability. It analyzes the role and expectations of actors that support this transition, as well as the expectations of the young people themselves and those of their families. A total of 32 students and 12 of their mothers were interviewed, in addition to teachers and school principals. The analysis of these interviews reveals that, regardless of their levels of achievement, all of the students expect to complete secondary education and successfully join the labor market. The adult references for these students are almost exclusively their families, and their accounts make no mention of actors from the school. Meanwhile, principals and teachers have low expectations regarding the educational future of the eighth-grade students and believe it to be a level of education on which they have little potential impact. In this context, students experience the transition to secondary education with little support from the schools, which, in addition to the low expectations, jeopardizes the success of this transition.
\end{abstract}

Keywords: Educational transitions; educational trajectories; primary education; secondary education; aspirations.

\title{
Expectativas en el proceso de transición en las trayectorias educativas
}

La trayectoria escolar se compone de distintos niveles de enseñanza, como la educación preescolar, primaria y secundaria. En la medida en que un estudiante avanza en su escolarización, atravesará etapas, eventos y transiciones que serán determinantes de su trayectoria educativa y que pueden tener impacto en otros dominios (Bernardi, Huinink, \& Settersten, 2019). Es posible comprender estos procesos desde la perspectiva de las trayectorias vitales o enfoque del curso de vida (Blanco, 2011). Desde este enfoque, la trayectoria vital se entiende como la yuxtaposición de factores biológicos, psicológicos, sociales y culturales que actúan de forma interdependiente, interactiva y acumulativa (Hutchison, 2019). Estas numerosas interacciones implican, a su vez, múltiples posibles caminos recorridos por los sujetos. Este enfoque pone hincapié en cómo la trayectoria vital de un individuo constituye una unidad en la cual lo ocurrido en un periodo de experiencia será determinante para la trayectoria posterior (Hutchison, 2019).

La investigación advierte acerca de la fuerte vinculación entre las experiencias de vida de las personas, los marcos institucionales en que estas se desenvuelven y los contextos sociohistóricos específicos que condicionan este proceso (Sepúlveda, 2013). Esto supone reconocer que las trayectorias se ven afectadas por las estructuras macro y la capacidad de acción individual en el contexto de las experiencias biográficas (Heinz \& Krüger, 2001). Así, desde el estudio de las trayectorias (Tilleczek, 2008), se observa cómo los sujetos construyen el sentido de sus vidas a partir de los distintos cambios en sus experiencias. En este contexto, las trayectorias escolares forman parte de las múltiples experiencias que componen el curso de la vida y que, por lo tanto, se ven influidas por factores de diversa naturaleza (Newman, 2020).

Durante el curso de vida de un sujeto, los diferentes dominios del espectro social conforman sus propias trayectorias, las cuales pueden yuxtaponerse unas a otras, así como analizarse de forma separada. En las sociedades actuales, el dominio de la educación se encuentra altamente institucionalizado, de manera tal que 
ordena la experiencia de los sujetos. Específicamente, en el sistema educativo, se organiza a niños, niñas y jóvenes en etapas o niveles de enseñanza diferenciados por grupos etarios y objetivos de enseñanza. Así, todos quienes estén dentro del sistema escolar deberán cursar progresivamente distintos niveles y vivir procesos de transición dentro de sus trayectorias educativas.

\section{Transiciones dentro de la trayectoria educativa}

Las transiciones refieren a cambios que afectan el curso de la vida de un sujeto, los que pueden originarse en su desarrollo (cambios biológicos o psicológicos) o en aspectos contextuales (cambios sociales o culturales). En este sentido, las transiciones consisten en la secuencia de cambios en el transcurso de la vida en relación con variables que afectan la trayectoria vital (curso de vida) de los sujetos (Billett et al., 2012; Wingens \& Reiter, 2012; Newman, 2020). La adolescencia es un periodo particularmente marcado por la secuencia de profundos cambios que afectan a los individuos, lo que deriva en una serie de transiciones críticas (Newman, 2020; Tilleczek $\&$ Ferguson, 2007). Entre ellas, las transiciones educativas propias del avance a través de la estructura de los niveles de enseñanza. Esta transición ocurre en yuxtaposición con otras actividades o transiciones que forman parte de la trayectoria de vida del estudiante y que, por lo tanto, tienen influencia en su definición como sujeto (Tilleczek, 2008). Específicamente, el paso de la educación primaria a la secundaria tiene un efecto persistente en el curso de la vida posterior en cuanto a los roles, estatus o identidades que asumen en relación con su entorno (Jindal-Snape, Symonds, Hannah, \& Barlow, 2019; Rogoff, Turkanis, \& Bartlett, 2001). Desde una perspectiva de desarrollo individual, el paso de un nivel educativo a otro tiene un impacto sobre el desarrollo social de las personas, su visión de sí mismas y su capacidad para adquirir nuevas habilidades y conocimientos (Colley, 2009). Al respecto, se ha señalado que una positiva promoción de las transiciones educativas permite a los estudiantes una mejor adaptación en relación con los docentes, pares, contenidos y metodologías de sus nuevos ambientes, promoviendo tanto el desarrollo de aprendizajes, habilidades y competencias sociales, como la mejora de los resultados educativos (Brooker, 2008).

\section{Expectativas de los adultos y la escuela frente al proceso de transición de los estudiantes}

El estudio de transiciones desde la educación primaria a la secundaria se ha concentrado en la experiencia de los propios estudiantes y los factores que influyen en el proceso (Jindal-Snape et al., 2019; Tilleczek \& Ferguson, 2007). Se hace énfasis en la diferencia entre la cultura primaria y cultura secundaria (Hargreaves, Earl, Moore, y Manning, 2001) y los factores que pueden determinar una trayectoria académica positiva de los estudiantes. Entre estos últimos, destacan la relación docente-estudiante, las relaciones con los pares, las creencias personales y factores emocionales (Escudero, González, y Martínez, 2009; Monarca, Rappoport, y González, 2012; Rodríguez Montoya, 2016; Jindal-Snape \& Cantali, 2019). Distintos autores afirman la importancia de la autopercepción académica en las trayectorias, junto con las expectativas de los propios estudiantes (Eccles \& Roeser, 2009; Jindal-Snape \& Miller, 2009, Jindal-Snape \& Cantali, 2019). En ese sentido, serán los padres quienes contribuyan a configurar las expectativas de los hijos en sus primeros años de trayectoria académica (Sewell, Hauser, Springer, \& Hauser, 2003); posteriormente, otros adultos de referencia o significativos irán configurando las expectativas educativas de los estudiantes (Lerner et al., 1996).

Los profesores poseen un rol clave respecto de la cultura escolar (Flores y Marini, 2019); por ello, las expectativas de los docentes son un factor que incide en diversos niveles del desarrollo del aprendizaje (Makuc, 2008). Los estudios revisados se han concentrado en las experiencias de los alumnos, o bien, han centrado sus esfuerzos en develar aquellas características del sistema de educación secundario que se deben mejorar para acompañar de mejor manera el proceso de transición (Jindal-Snape, Cantali, MacGillivray, \& Hannah, 2019). Por ende, las investigaciones realizadas coinciden en que las diferencias entre ambos niveles deben ser trabajadas para aminorar 
las consecuencias negativas de los cambios que conlleva este proceso. No obstante, no se han enfocado en dar cuenta de la influencia de las expectativas, percepciones y creencias de las escuelas en este periodo, a pesar de que se reconocen como factores clave. De este modo, indagar en las expectativas y discursos de aquellos adultos que pueden ser una referencia para los estudiantes explora una arista del proceso de transición que complementa las perspectivas anteriormente estudiadas respecto de la temática.

\section{Transición desde la educación primaria a la educación secundaria en Chile}

En Chile, la Ley General de Educación ( $N^{\circ}$ 20.370) establece la obligatoriedad de 12 años de nivel educacional, lo que ha incrementado los niveles de cobertura de la educación en el territorio. Ello implica que existe una obligación de cursar la educación básica y media, siendo también mandatorio el realizar un proceso de transición entre ambos niveles. Específicamente, el paso de la educación básica a la media ha sido diagnosticado como una de las transiciones más críticas del sistema escolar chileno (Dávila, Ghiardo, y Medrano, 2005; Raczynski, Hernández, Kegevic, y Rocco, 2011), teniendo consecuencias negativas en el rendimiento académico y en las tasas de deserción de estudiantes (Treviño, Villalobos, Hernández, Vielma, y Valenzuela, 2016). Estudios como el realizado por Hernández y Raczynski (2014) resaltan también el contexto de los alumnos del sector rural y de los estudiantes más vulnerables del sistema educativo. A estos antecedentes cabe agregar que, cuando se habla de vulnerabilidad, se refiere a condiciones que afectan no solo los resultados escolares, sino que también implican un menor acceso a la atención médica, la cultura, la recreación y, en general, a una menor calidad de vida (Yoshikawa, Aber, \& Beardslee, 2012). En este sentido, los resultados de la prueba SIMCE indican no solo un descenso de los resultados académicos de los estudiantes a lo largo de su trayectoria educativa, que se intensifica en los grupos socioeconómicos (GSE) más bajos, sino que además los indicadores de "autoestima y motivación” siguen la misma tendencia (Agencia de Calidad de la Educación (ACE), 2017).

En este contexto, factores como las expectativas de los estudiantes y de los adultos que acompañan el proceso pueden ser determinantes para el curso de este, tanto para dar continuidad a una trayectoria, limitarla o transformarla. Este artículo tiene como objetivo el estudio de un momento específico de la trayectoria educativa de un grupo de vulnerable de estudiantes de la educación municipal en Chile. Se observará específicamente cómo se despliegan las expectativas de los actores (directivos, docentes, estudiantes y sus familias) que siguen el curso de vida y acompańan la transición desde la educación primaria a la secundaria. Los resultados que se presentan en este

artículo se generaron en el marco de un estudio sobre factores determinantes del éxito académico y de las trayectorias de estudiantes de octavo grado de contextos vulnerables en la región de O’Higgins (Gómez y Rivas, 2017).

\section{Metodología}

En términos generales, se han desarrollado procedimientos cuantitativos y cualitativos en etapas sucesivas y con recurrencias. En consecuencia, el diseño de investigación es mixto, ya que involucró la recolección y análisis de datos cuantitativos relevantes y estudios de caso considerando una variedad de informantes (Denzin \& Lincoln, 2005; Woodside, 2010; Teddlie \& Tashakkori, 2009). 


\section{Participantes}

Para la identificación de la muestra se utilizó el índice IVE-Sinae, que mide la vulnerabilidad escolar del establecimiento de acuerdo con la proporción de estudiantes en riesgo social en los establecimientos educacionales del país. Tal como lo presenta la tabla 1, el porcentaje de vulnerabilidad en la sexta región se aproxima a la media nacional, lo que es significativo, puesto que se trata de una región poco estudiada hasta ahora ${ }^{1}$.

Tabla 1

Comparación del indice de vulnerabilidad de los establecimientos escolares chilenos 2017 según media regional y nacional.

\begin{tabular}{|c|c|c|c|}
\hline & Región de O'Higgins & Región Metropolitana & Media nacional \\
\hline $\begin{array}{l}\text { Vulnerabilidad en establecimientos } \\
\text { de educación básica }\end{array}$ & $78 \%$ & $70 \%$ & $80 \%$ \\
\hline
\end{tabular}

Fuente: Elaboración propia. Cálculo desarrollado con base en datos oficiales de establecimientos educacionales 2017.

Para concentrar la muestra en establecimientos de alta vulnerabilidad, se calculó el 25\% superior del índice IVE-SINAE en la región (>89\%). La muestra consideró establecimientos de dependencia municipal y se buscó mantener una proporción respecto de las provincias que constituyen la división administrativa de la región. 127 establecimientos cumplían con estas características para el ańo escolar 2017; no se consideraron en esta muestra establecimientos cuya matrícula fuese particularmente pequeña ( $>15$ alumnos matriculados). A partir de ellos, se generó un grupo de seis establecimientos (dos de cada provincia, tres de ellos rurales) que constituyeron la muestra para el estudio. A nivel individual, la muestra se compuso por 192 estudiantes de octavo ańo básico.

Tabla 2

Caracterización de los establecimientos participantes en el estudio.

\begin{tabular}{cccccc} 
& Provincia & Urbano/Rural & $\begin{array}{c}\text { Matrícula total } \\
2017\end{array}$ & $\begin{array}{c}\text { Matricula } \\
\text { octavo básico }\end{array}$ & Ive-Sinae 2017 \\
\hline Caso 1 & Cachapoal & Urbano & 293 & 20 & 0,91 \\
\hline Caso 4 & Cachapoal & Urbano & 558 & 58 & 0,88 \\
\hline Caso 2 & Cardenal Caro & Rural & 56 & 3 & 0,88 \\
\hline Caso 3 & Cardenal Caro & Rural & 110 & 9 & 0,96 \\
\hline Caso 5 & Colchagua & Rural & 171 & 18 & 0,88 \\
\hline Caso 6 & Colchagua & Urbano & 713 & 0,88 \\
\hline
\end{tabular}

Fuente: laboración propia.

1. Gran parte de la investigación en educación en esta temática en Chile toma a la Región Metropolitana como referencia, o no hace distinción entre esta y las demás. La menor proporción de vulnerabilidad de la Región Metropolitana podría explicarse por la mayor proporción de establecimientos educacionales que atienden a la élite económica del país. 


\section{Procedimientos}

En cada establecimiento se aplicaron cuestionarios para indagar respecto de trayectorias, transiciones, factores protectores y de riesgo asociados a ella. En el cuestionario también se incorporaron preguntas para conocer las características del grupo familiar, su composición, nivel de ingresos y oficios de ambos padres. Para la evaluación del rendimiento en comprensión lectora se utilizó la Prueba de Comprensión Lectora de Complejidad Lingüística y Progresiva (CLP), instrumento estandarizado y validado en Chile y ampliamente conocido por los docentes del sistema escolar. En una segunda fase del estudio, se llevaron a cabo entrevistas en profundidad con 32 estudiantes de acuerdo con la distribución de su rendimiento en la prueba CLP: 12 estudiantes de alto rendimiento, 12 estudiantes de rendimiento medio y ocho estudiantes de bajo rendimiento. De este grupo, se entrevistó a las madres de 12 estudiantes, cinco de estudiantes de alto rendimiento, cinco de estudiantes de rendimiento regular y dos de rendimiento bajo. Se entrevistaron también cinco directores de los establecimientos participantes y cinco docentes de Lenguaje y Comunicación de cada curso del establecimiento cuyos alumnos participaron del estudio (ver apéndice: pautas de entrevista).

La investigación de terreno se desarrolló entre agosto y noviembre de 2017; en primer lugar, se invitó a participar a los directores de los establecimientos, quienes facilitaron el contacto con los docentes de octavo grado, los padres y estudiantes. Todos los participantes fueron informados respecto de los objetivos de la investigación, sus derechos como participantes y el compromiso de confidencialidad sobre la información recolectada. Todos los participantes firmaron formularios de consentimiento informado, previamente validados por el comité de ética de la universidad patrocinante. Las entrevistas fueron realizadas en las escuelas, en oficinas facilitadas por la administración.

Los resultados de este artículo se focalizan en un análisis temático (Braun \& Clarke, 2006) de las entrevistas semiestructuradas aplicadas. El proceso de análisis se desarrolló mediante doble codificación con base en temas de interés para el objetivo de estudio (expectativas sobre los estudiantes, imagen sobre los actores, rol de la escuela y rol de la familia). Tras un primer proceso de codificación con el software Nvivo 11, el equipo de análisis comparó y ajustó las codificaciones para aumentar el nivel de acuerdo alcanzado (Kappa 0,84). Posteriormente, se procedió a codificar la totalidad de los datos. Se presentan a continuación los resultados del análisis temático de las entrevistas.

\section{Resultados}

\section{Discurso de estudiantes sobre la transición y los agentes de apoyo}

Dentro de las expectativas, el análisis se centró en las ambiciones académicas de los estudiantes, consultando por sus proyecciones inmediatas y futuras. Estas fueron categorizadas de acuerdo a si se referían al nivel secundario o al terciario, respectivamente. Respecto de las proyecciones inmediatas, el curso octavo básico es el último de la educación primaria en Chile, desde el año 2017; para matricularse en el siguiente curso, las familias postulan a tres establecimientos escolares de educación secundaria, a los cuales son asignados en forma aleatoria. En este contexto, durante el período de levantamiento de información, los jóvenes se encontraban en proceso de postulación y manifestaron cierta incertidumbre respecto del siguiente año: "Voy a ver en qué liceo quedo, porque como ahora es por Internet, no es el liceo que uno quiere" (Est. Caso $2^{2}$ ). Respecto del proceso de postulación, no se realizaron preguntas explícitas de valoración de este en las entrevistas. Sí se preguntó a los jóvenes a qué liceos estaban postulando y cuáles eran sus expectativas sobre los establecimientos secundarios. $\mathrm{Al}$ respecto, en general -y sin distinción por el rendimiento académico alcanzado por los estudiantes en la prueba de lectura- se mostraban optimistas de continuar la educación secundaria. Sin embargo, y a pesar de

2. Est. = estudiante, $\mathrm{E}=$ Entrevistador; $\mathrm{D}=$ Director, $\mathrm{P}=$ Profesor, $\mathrm{A}=$ Apoderado. 
la importancia que la literatura otorga al acompañamiento de los adultos que componen la comunidad escolar, en sus narraciones sobre el proceso de postulación no se mencionaron adultos, ni familiares ni personal de la escuela, que hubiesen apoyado o facilitado el proceso.

La enseñanza secundaria chilena se divide en dos grandes ramas: los liceos humanista-científico, cuya meta es la preparación para cursar estudios universitarios, y los liceos técnico-profesionales, que entregan una especialización laboral al finalizar los cuatro años de estudios secundarios. Del conjunto de estudiantes entrevistados, respecto de sus proyecciones futuras, solamente dos (pertenecientes al grupo de mayor rendimiento en la prueba de lectura) declararon explícitamente optar por la educación humanista-científica para luego continuar estudios universitarios. La mayor parte de los jóvenes declaró su preferencia por los estudios técnico-profesionales, que les permitirían obtener una profesión. Entre sus argumentos para optar por los liceos técnicos está la necesidad de mejorar sus condiciones materiales y simbólicas en el futuro: "Así puedo llegar a ser alguien en la vida, puedo ser alguien; no voy a ser alguien que no tenga nada, no tenga profesión, no tenga buen estar" (Est. caso 1); "Si no estudio algo, me quedaría sin nada. Aparte que, en tercero medio, ya uno tiene que elegir su especialidad en ese liceo. Entonces, si no estudio, no sé qué haría" (Est. caso 2). Los jóvenes también ponen énfasis en aumentar los aprendizajes que han alcanzado hasta ahora: "Si no me va bien ahora, imposible que me vaya bien en el futuro si acá no aprendí lo necesario" (Est. caso 6). Solo hubo dos casos en los que se veía a la educación secundaria como la última etapa de sus estudios y se optaba por otras metas, como ser futbolista profesional o postular a la academia policial o militar; esto coincide con estudiantes de menor rendimiento en la prueba de lectura.

Estas decisiones tenían, en varios casos, referencias en la familia de los estudiantes tanto para la elección del establecimiento como para las especialidades a seguir: "Yo tengo un hermano... a él le ha ido bien en ese liceo, va a salir de cuarto medio [pausa]; él ha hecho varias cosas con fierro, una metralleta, un helicóptero" (Est. caso 2); "E: ¿Por qué te gusta mecánico? A: Porque mi papá, mi primo, mi hermano, mi tata ${ }^{3}$, son mecánicos."(Est. caso 6); "Igual me quiero ir al [nombre de establecimiento], porque igual mi mami estuvo ahí" (Est. caso 5). En ninguna de las entrevistas los estudiantes mencionaron a docentes u otros profesionales de sus escuelas como referencias o informantes respecto de sus elecciones para la educación secundaria.

En otro punto de la entrevista, se pidió a los jóvenes hablar de los adultos a quienes admiran, específicamente de su entorno familiar y escolar. Como ya se ha señalado, en el contexto de la transición, un factor clave es la presencia de adultos que funcionan como referentes para los jóvenes. En sus discursos, sin distinción de resultados en la prueba de lectura, los estudiantes declararon admirar a sus madres, padres o ambos. Por ejemplo, un estudiante refiriéndose a sus padres dijo admirarlos porque "son como una figura más grande que yo, quiero ser como ellos" (Est. caso 6); en otro caso, una estudiante declaró admirar a su madre porque

Ella ha sabido sacarme adelante sola, sin la ayuda de nadie. E: Y de otros adultos que tú conozcas, aparte de tu familia, ¿alguien más que admires? Est: [Duda] No. E: ¿¿Solo tu mamá? Est: Es la heroína (Est. caso 6).

Al solicitarles mencionar a un segundo adulto a quien admiraran, se mencionan otros adultos de la familia como abuelos, tíos o hermanos. Los profesores, por su parte, solo fueron mencionados como adultos admirados en tres casos, y solo ante esta segunda pregunta de profundización, nunca en primer lugar o como eje de la narración.

Frente a esta evidencia, nos preguntamos qué indican los discursos de los directivos y docentes que acompañan el proceso de transición respecto de las expectativas académicas futuras de estos jóvenes y cuál es el rol que desempeñan.

3. Abuelo en lenguaje coloquial chileno. 


\section{Expectativas de directivos y docentes ante la transición}

Sobre las expectativas académicas que los directivos de los establecimientos tienen sobre los estudiantes en transición, se observa que están principalmente determinadas por los resultados académicos de los estudiantes, ya sea por las calificaciones o por los resultados obtenidos en pruebas estandarizadas. En este contexto, el SIMCE (sistema de medición de calidad de la educación en Chile) es un referente recurrente de la narración: "D: En octavo, también estamos al alza, más bajo que en cuarto, pero también estamos al alza en relación con la comprensión lectora. Hemos subido varios puntos" (D. Caso 2); "Tenemos la esperanza de que ese curso, por lo menos, supere la media nacional... Es como un desafío para el curso. E: ¿Y eso ellos lo asumen así? D: Ellos lo asumen así" (D. Caso 4). Al usar los resultados SIMCE para fundamentar sus afirmaciones respecto de la calidad académica de los estudiantes, se infiere que el relato se construye considerando que los resultados de estas evaluaciones son determinantes en el éxito de las trayectorias académicas.

Por otra parte, el discurso de los directores es el que presenta mayores dudas respecto del desarrollo académico de los estudiantes que inician la transición:

Los cuartos, los terceros, son cursos que están bastante motivados con el tema de las lecturas. E: ¿Y los octavos y los séptimos? D: Los octavos y los séptimos ha costado... aunque tenemos la esperanza de que ese curso, por lo menos, supere la media nacional (D. Caso 2).

En otro caso, una directora declara: "Octavo es un curso bastante potenciable, pero depende de la motivación, si ellos se entregan a dar un buen resultado o no (...) Entonces no les va mal, yo no creo que les vaya a ir tan mal” (D. Caso 1).

Llama la atención que los directores afirmen que los estudiantes pueden enfrentar dificultades académicas en la educación secundaria sin, al mismo tiempo, dar cuenta de una responsabilización respecto de este mismo hecho: "(...) Algunos desertan en primero o segundo medio porque encuentran exigencia... porque las bases con que llegan no son las más sólidas" (D. Caso 2); un caso específico es el de un establecimiento que cuenta con un programa vespertino de regularización de estudios y que relata positivamente la frecuencia con la que estudiantes egresados de la educación básica en el mismo establecimiento vuelven a completar su educación media en la nocturna: “(...) Lo que pasa es que, a la vuelta del camino, estos niños que fracasan en la educación diurna llegan a nuestras nocturnas y terminan con nosotros cuarto medio... Terminan, por distintas vías acá... Es como volver a su casa" (D. Caso 1). El relato de la directora de este establecimiento no establece una relación entre el fracaso en la educación secundaria y la labor pedagógica desarrollada en la educación primaria.

El discurso de los docentes alude con menor frecuencia al rendimiento académico; en su lugar, se habla frecuentemente de aspectos emocionales de los jóvenes, de dificultades para continuar por falta de interés, de estudiantes que son perezosos o de la falta de acompañamiento (no tienen apoyo del hogar) o de referentes familiares: "Nosotros tenemos un 100\% de apoderados de campo... se ha hecho un trabajo para sacarlos un poquito de este contexto... que no necesariamente el trabajo está en el campo" (P. Caso 4). Ante ello, la reacción de los docentes es reforzar discursivamente, por medio de diálogos, la motivación de los jóvenes: "Hemos conversado mucho, o sea, se han hecho intervenciones con los dos octavos" (P. Caso 2); "Nosotros tenemos mucha esperanza de muchos alumnos; de hecho, a todos les decimos que son capaces" (P. Caso 1); "Trato de mostrarles que yo confío en ellos, de la misma forma que lo hacen el director y todos los profesores" (P. Caso 4).

En este contexto, el discurso de los docentes es igualmente de bajas expectativas; ante la pregunta por el futuro desempeño académico de los jóvenes, las respuestas tienden a relativizar sus posibilidades: "E: ¿Y cómo espera que le vaya este año al octavo académicamente? P: Creo que no les va a ir brillante [pausa], pero tampoco mal" (P. Caso 6); "E: Y en general, como curso, ¿̨cómo crees que les va a ir al cierre del ańo? P: [tenemos] dos alumnos que pueden repetir [pausa]. Pero los otros tampoco son tan excepcionales" (P. Caso 1). 


\section{El rol de la escuela en la transición}

De acuerdo con el análisis de los datos, se relevaron dos categorías referidas al rol de las familias y al de los agentes educativos en la trayectoria educativa de los nińos. Las familias de todos los estudiantes ven en la escuela una figura de formación para los nińos, niñas y jóvenes. Dado su contexto de vulnerabilidad y la precariedad de las condiciones en que se desenvuelven, los familiares no pueden prestar un apoyo académico sistemático a los estudiantes (ya sea por carencias de tiempo, conocimientos para el apoyo de los quehaceres académicos, etc.). Por ende, delegan la tarea de la compañía en esta trayectoria a la escuela y sus actores. Así, en la mayoría de los casos, no existen estrategias para el reforzamiento académico de los estudiantes en las casas o la promoción de ciertas habilidades o conocimientos. En el caso de los familiares que reconocen que el estudiante tiene buenas calificaciones, existe una preocupación de la familia por apoyarlo en el estudio o la realización de quehaceres escolares.

Por otro lado, desde el discurso de los actores de la escuela se desprende que no existen estrategias que apunten a la motivación de los estudiantes de octavo grado; más bien, los programas apuntan a los primeros ciclos de enseñanza primaria. Ahora bien, en el caso de existir estrategias, están enfocadas en el mejoramiento de indicadores del establecimiento educacional, como asistencia o puntajes obtenidos en la prueba SIMCE:

(...) Hemos estado trabajando la autoestima, diciéndoles de todos estos beneficios por la clasificación del colegio, explicándoles lo que es, que ellos tienen que dejar un legado para sus compañeros, porque ellos ya recibieron beneficios que venían de otros triunfos anteriores (D. Caso 1).

Junto con ello, los actores de las escuelas de la muestra señalan que han desistido de trabajar en conjunto con las familias, debido a la baja participación o porque hacerlo implica dificultades para ellos:

Y tuvimos que aprender, y es una fortaleza, a trabajar sin el apoyo del apoderado, el nińo aprende las ocho horas que está acá. Escasa tarea al hogar, porque sabemos que o no tiene los medios o no tiene quien le ayude (D. Caso 1).

En consecuencia, el trabajo con estudiantes y familias, y el enfoque de estas acciones en conjunto, dependerán de la gestión del director o directora a cargo, quien puede cambiar cada cuatro ańos. El cambio en el cargo, en muchos casos, significa que el director tendrá como principal preocupación trabajar con los estudiantes de primer ciclo, para así "partir con ellos desde cero" (P. Caso 4). Por otro lado, las iniciativas que pueden tener los docentes para el trabajo con los estudiantes de estos niveles no pueden generar mayor impacto, pues no son parte de un proyecto institucional.

\section{Conclusiones}

En este estudio se ha descrito la importancia de la transición desde la educación primaria a la secundaria en el marco de las trayectorias educativas de los jóvenes participantes. La revisión de literatura puso en relieve hasta qué punto una transición positiva puede significar un cambio en la trayectoria educativa de los jóvenes. Esto permitió, a su vez, subrayar el impacto que pueden tener las comunidades educativas que acompañan un proceso de transición sobre las trayectorias de vida. Frente a este escenario, se vuelve relevante observar a los estudiantes que se encuentran ad-portas de transitar desde la educación primaria a la secundaria. Nos hemos centrado específicamente en las expectativas que los estudiantes tienen sobre su futuro académico y en el correlato generado por los directivos, docentes y sus familias, así como en el rol que asume la institución escolar respecto del proceso. 
Se observa que las expectativas analizadas son parte de una transición educativa altamente ordenada por la normativa legal, y que para el caso de la educación municipal en Chile contempla, con escasas excepciones, el cambio físico desde una institución educativa primaria hacia otra institución educativa secundaria. Respecto del grupo social observado, nos encontramos frente a una población altamente vulnerable en términos económicos y sociales, pero también en lo concerniente a su zona geográfica, cercana al contexto agroindustrial.

Sobre las expectativas de los estudiantes en transición se pudo observar que, sin diferencias por sus rendimientos en la prueba de lenguaje aplicada, todos los estudiantes tienen expectativas positivas sobre su avance hacia la educación secundaria, incluso proyectándose en su educación terciaria y futuro laboral. Respecto de esto último, resulta interesante la importancia que adquiere en sus narraciones el tipo de educación secundaria que recibirán. En este sentido, gran parte de los entrevistados esperan transitar hacia la educación técnico-profesional para obtener una cualificación que, tras el egreso de la educación secundaria, les permita incorporarse al mundo laboral. Este hallazgo dota de una relevancia aún mayor al proceso de transición desde la educación primaria a la secundaria en este grupo de jóvenes y a la pregunta sobre el rol que en ella juegan sus comunidades educativas, sobre todo si se considera que para ellos la referencia fundamental y casi exclusiva de proyección en la educación secundaria son sus familiares cercanos, con casi total ausencia de referencias a los actores del entorno escolar.

Específicamente, las expectativas de los adultos respecto del éxito de los jóvenes en su trayectoria educativa es un factor relevante y destacado en la literatura. Sin embargo, este estudio demostró que directivos y profesores de establecimientos que atienden a estudiantes de alta vulnerabilidad tienen bajas expectativas respecto del futuro educativo de los estudiantes de octavo grado. Desde las narraciones de directivos y docentes, se cuestiona el éxito de las trayectorias académicas de los estudiantes. Los directivos aluden más frecuentemente a los resultados de evaluaciones estandarizadas como referencia de relativización del posible éxito. Los docentes, por su parte, aluden a factores emocionales fuera de lo académico. En sus narraciones, los docentes tienen expectativas medias o bajas respecto del desarrollo académico futuro de los estudiantes. Esto se profundiza por la visión desde los establecimientos del octavo grado como un nivel en el que las posibilidades de impacto de las políticas de la escuela básica serían reducidas.

Respecto del rol de la familia, los relatos dan cuenta de una desconexión entre la experiencia de los estudiantes y la experiencia educativa escolar. Mientras que la familia es el pilar referencial para los jóvenes, los apoderados confían en el rol de la escuela. Directivos y docentes, por su parte, niegan el rol de la familia al considerarla como no relevante para la experiencia escolar de los jóvenes. Esta visión se asocia frecuentemente al carácter rural y de vulnerabilidad de las familias.

En consecuencia, el análisis sobre los roles de agentes evidencia que para los estudiantes de octavo grado tanto la familia como la escuela se desentienden de su acompañamiento académico en la transición. En el caso de las familias, esto ocurre no por falta de interés, sino que por falta de recursos académicos que permitan apoyar a los estudiantes. Por otra parte, las escuelas observan a los octavos grados como un nivel en el que tienen poca injerencia, prefiriendo destinar sus recursos a los niveles anteriores. Esta situación genera un vacío en el espacio social escolar, en donde el énfasis está puesto en la agencia de los estudiantes como principal motor para el desarrollo de trayectorias escolares positivas.

En los casos de estudio, no se constata la existencia de una política o proyecto institucional que incentive el soporte de los jóvenes durante la transición a la enseńanza media. El apoyo hacia los estudiantes queda supeditado a las iniciativas personales de los adultos que los acompañan, cuyas acciones no son coordinadas. En este contexto, se pone en riesgo el éxito de la transición y, en consecuencia, del desarrollo positivo de las trayectorias de los jóvenes. Parte de este proyecto debiese incorporar a la familia, dado su rol referencial para las trayectorias de los 
jóvenes. En este sentido, es posible hipotetizar que un trabajo que fomente la confluencia entre esta y la escuela podría tener un impacto positivo en las trayectorias, mejorando el estado actual del proceso de acuerdo con los resultados de este estudio y su vinculación con la literatura que aborda esta temática.

El artículo original fue recibido el 3 de octubre de 2019

El artículo revisado fue recibido el 1 de abril de 2020

El artículo fue aceptado el 18 de junio de 2020

\section{Referencias}

Agencia de Calidad de la Educación. (2017). Factores asociados a los resultados educativos 2017. Santiago de Chile: MINEDUC.

Bernardi, L., Huinink, J., \& Settersten Jr, R. A. (2019). The life course cube: A tool for studying lives. Advances in Life Course Research, 41, 100258. https://doi.org/10.1016/j.alcr.2018.11.004

Billett, S., Johnson, G., Thomas, S., Sim, C., Hay, S., \& Ryan, J. (Eds.). (2012). Experience of school transitions: Policies, practice and participants. Springer Science \& Business Media.

Blanco, M. (2011). El enfoque del curso de vida: orígenes y desarrollo. Revista Latinoamericana de Población, 5(8), 5-31. https://doi.org/10.31406/relap2011.v5.i1.n8.1

Braun, V. \& Clarke, V. (2006). Using thematic analysis in psychology. Qualitative research in psychology, 3(2), 77-101. https://doi.org/10.1191/1478088706qp063oa

Brooker, L. (2008). Supporting transitions in the early years. Reino Unido: McGraw-Hill Education.

Colley, H. (2009). Time in learning transitions through the lifecourse: A feminist perspective. En K. Ecclestone, G. Biesta, \& M. Hughes (Eds)., Transitions and learning through the Lifecourse (pp. 154-170). Nueva York, NY: Routledge.

Dávila, O., Ghiardo, F., y Medrano, C. (2005). Los desheredados. trayectorias de vida y nuevas condiciones juveniles. Valparaíso, Chile: Centro de Investigación y Difusión Poblacional de Achupallas.

Denzin, N. K. \& Lincoln, Y. S. (Eds.). (2005). The Sage handbook of qualitative research. Londres, Reino Unido: Sage.

Eccles, J. S. \& Roeser, R. W. (2009). Schools, academic motivation, and stage-environment fit. En R. M. Lerner \& L. Steinberg (Eds.), Handbook of adolescent psychology, 1 (pp. 404-434). Hoboken.

Escudero, J. M., González, M. T., y Martínez, B. (2009). El fracaso escolar como exclusión educativa: comprensión, políticas y prácticas. Revista iberoamericana de educación, 50, 41-64. https://doi.org/10.35362/rie500660

Flores, L y Marini, G. (2019). Dimensiones olvidadas de la reforma educativa hoy: la experiencia del espacio y el lugar en la escuela. En A. Carrasco y L. M. Flores (Eds.). De la reforma a la transformación: capacidades, innovaciones y regulación de la educación chilena (pp. 241-264). Santiago, Chile: Ediciones UC.

Gómez, G. y Rivas, M. (2017). Resiliencia académica, nuevas perspectivas de interpretación del aprendizaje en contextos de vulnerabilidad social. Calidaden la educación, (47), 215-233. https://doi.org/10.4067/S0718-45652017000200215

Hargreaves, A., Earl, L., Moore, S., y Manning, S. (2001). Aprender a cambiar. La enseñanza más allá de las materias y los niveles. Barcelona, España: Octaedro.

Heinz, W. R. \& Krüger, H. (2001). Life course: Innovations and challenges for social research. Current sociology, 49(2), 29-45. https://doi.org/10.1177/0011392101049002004

Hernández, M. y Raczynski, D. (2014). Jóvenes de origen rural: aspiraciones y tensiones en la transición hacia la enseñanza secundaria. Revista Iberoamericana de Evaluación Educativa, 7(3), 71-87. Recuperado de http://hdl.handle.net/20.500.12799/3320

Hutchison, E. (2019). An update on the relevance of the life course perspective for social work. Families in Society, 100(4), 351-366. https://doi.org/10.1177/1044389419873240 
Jindal-Snape, D. \& Cantali, D. (2019). A four-stage longitudinal study exploring pupils' experiences, preparation and support systems during primary-secondary school transitions. British Educational Research Journal, 45(6), 1255-1278. https://doi.org/10.1002/berj.3561

Jindal-Snape, D., Cantali, D., MacGillivray, S., \& Hannah, E. (2019). Primary-Secondary Transitions: A Systematic Literature Review. (Social Research Series). Escocia: Scottish Government.

Jindal-Snape, D. \& Miller, D. J. (2009). Understanding transitions through self-esteem and resilience. En D. JindalSnape (Ed.), Educational transitions (pp. 11-32). Nueva York, NY: Routledge.

Jindal-Snape, D., Symonds, J., Hannah, B., \& Barlow, W. (2019, December). Conceptualising primary to secondary school transitions: A systematic literature review. En School of Education and Social Work Research and Scholarship Conference 2019.

Lerner, R. M., Lerner, J. V., von Eye, A., Ostrom, C. W., Nitz, K., Talwar-Suni, R., \& Tubman, J. G. (1996). Continuity and discontinuity across the transition of early-adolescence: A developmental contextual perspective. En J. A. Garber, J. Brooks-Gunn, \& A. C. Petersen (Eds.), Transition through adolescence: Interpersonal domains and context (pp. 3-22). Mahwah, NY: Earlbaum.

Makuc, M. (2008). Teorías implícitas de los profesores acerca de la comprensión de textos. Revista signos, 41(68), 403-422. https://doi.org/10.4067/S0718-09342008000300003

Monarca, H., Rappoport, S., y González, A. F. (2012). Factores condicionantes de las trayectorias escolares en la transición entre enseñanza primaria y secundaria. Revista Española de Orientación y Psicopedagogía, 23(3), 49-62. https://doi.org/10.5944/reop.vol.23.num.3.2012.11461

Newman, D. M. (2020). Sociology: Exploring the architecture of everyday life. Sage.

Raczynski, D., Hernández, M., Kegevic, L., y Rocco, R. (2011). El paso de la enseñanza básica a la media en estratos bajos: Un reto a la igualdad de oportunidades educativas. Santiago, Chile: Ministerio de Educación. Recuperado de https://centroestudios.mineduc.cl/wp-content/uploads/sites/100/2017/07/Informe-Final-Dagmar-Raczynski-F511083.pdf

Rodríguez Montoya, F. M. (2016). Transición de primaria a secundaria: factores de éxito. (Tesis doctoral inédita). Universidad de Barcelona.

Rogoff, B., Turkanis, C. G., \& Bartlett, L. (2001). Learning together: Children and adults in a school community. Oxford, Reino Unido: Oxford University Press.

Sepúlveda, L. (2013). Juventud como transición: elementos conceptuales y perspectivas de investigación en el tiempo actual. Última década, 21(39), 11-39. https://doi.org/10.4067/S0718-22362013000200002

Sewell, W. H., Hauser, R. M., Springer, K. W., \& Hauser, T. S. (2003). As we age: A review of the Wisconsin Longitudinal Study, 1957-2001. Research in social stratification and mobility, 20, 3-111. https://doi.org/10.1016/S0276-5624(03)20001-9

Teddlie, C. \& Tashakkori, A. (2009). Foundations of mixed methods research: Integrating quantitative and qualitative approaches in the social and behavioral sciences. Londres, Reino Unido: Sage.

Tilleczek, K. (2008). Building bridges: Transitions from elementary to secondary school. Education Canada, 48(1), 68-71. Recuperado de https://eric.ed.gov/?id=EJ782702

Tilleczek, K. \& Ferguson, B. (2007, January). Fresh starts/false starts: A review of literature on the transition from elementary to secondary school. In Ontario Education Research Symposium.

Treviño, E., Villalobos, C., Hernández, C., Vielma, C., y Valenzuela, J. P. (2016). Trayectorias escolares de los estudiantes y agrupamiento al interior del aula en los colegios chilenos de enseńanza media. Análisis de la heterogeneidad académica al interior de las escuelas. Pensamiento Educativo, 53(2), 1-16. Recuperado de http://pensamientoeducativo.uc.cl/index.php/pel/article/view/25975

Wingens, M. \& Reiter, H. (2012). The life course approach-it's about time! BIOS-Zeitschrift für Biographieforschung, Oral History und Lebensverlaufsanalysen, 24(2), 9-10. Recuperado de https://elibrary.utb.de/doi/10.3224/bios.v24i2.05

Woodside, A. G. (2010). Case study research: Theory, methods and practice. Reino Unido: Emerald.

Yoshikawa, H., Aber, J. L., \& Beardslee, W. R. (2012). The effects of poverty on the mental, emotional, and behavioral health of children and youth: implications for prevention. American Psychologist, 67(4), 272-284. https://doi.org/10.1037/a0028015 


\section{Apéndice.}

Entrevistas en profundidad, temas y conceptos desarrollados

Tema

\begin{tabular}{|c|c|}
\hline \multirow{3}{*}{$\begin{array}{l}\text { La escuela como entorno } \\
\text { protector }\end{array}$} & Percepción de la escuela como espacio de protección \\
\hline & Percepción de vulnerabilidad en el entorno escolar \\
\hline & Actividades extracurriculares \\
\hline \multirow{4}{*}{$\begin{array}{c}\text { Imagen y expectativas sobre } \\
\text { los actores }\end{array}$} & Expectativas de logro académico de los niños \\
\hline & Imagen de padres en tanto que modelos de resiliencia \\
\hline & Imagen de otros adultos o pares en tanto que modelos de resiliencia \\
\hline & Trayectorias escolares \\
\hline \multirow{2}{*}{ Experiencias de resiliencia } & Situaciones de resiliencia a nivel familiar o individual \\
\hline & Experiencias positivas vividas a nivel familiar o individual \\
\hline \multirow{2}{*}{ Autoimagen académica } & Autopercepción de los niños en tanto que estudiantes \\
\hline & Estrategias de estudio para desafíos académicos \\
\hline \multirow{4}{*}{$\begin{array}{l}\text { Características } \\
\text { sociodemográficas }\end{array}$} & Condiciones de salud del niño o su familia \\
\hline & Condiciones habitacionales del niño y su familia \\
\hline & Entorno del espacio educativo \\
\hline & $\begin{array}{c}\text { Pertenencia minoría étnica, procesos migratorios, género, } \\
\text { grupo religioso, organizaciones sociales }\end{array}$ \\
\hline \multirow{2}{*}{ Tiempo } & Uso del tiempo a lo largo del último año \\
\hline & Uso del tiempo en el hogar \\
\hline \multirow{3}{*}{$\begin{array}{l}\text { Acceso y disponibilidad de } \\
\text { recursos }\end{array}$} & Disponibilidad de recursos materiales de estudio en el hogar \\
\hline & Acceso a la escuela \\
\hline & Disponibilidad de recursos y materiales en la escuela \\
\hline Antecedentes de docentes & $\begin{array}{c}\text { Perfil de los docentes (formación, años de experiencia, } \\
\text { cursos en que enseña, disciplinas) }\end{array}$ \\
\hline \multirow{2}{*}{ Antecedentes institucionales } & Políticas escolares para los estudiantes resilientes \\
\hline & Políticas escolares focalizadas para estudiantes vulnerables \\
\hline
\end{tabular}

